Transport and Communications Science Journal

\title{
AN APPROXIMATE FORMULA TO CALCULATE THE CRITICAL DEPTH IN CIRCULAR CULVERT
}

\author{
Hoang Nam Binh \\ University of Transport and Communications, No 3 Cau Giay Street, Hanoi, Vietnam
}

\author{
ARTICLE INFO \\ TYPE: Research Article \\ Received: $31 / 5 / 2020$ \\ Revised: $17 / 9 / 2020$ \\ Accepted: 18/9/2020 \\ Published online: 30/9/2020 \\ https://doi.org/10.47869/tcsj.71.7.9 \\ * Corresponding author \\ Email: binhhn@utc.edu.vn
}

\begin{abstract}
Critical depth is a depth of flow where a specific energy section is a minimum value with a flow rate. Critical depth is an essential parameter in computing gradually varied flow profiles in open channels and in designing culverts. If cross-sections are rectangular or triangular, the critical depth can be computed by the governing equation. However, for other geometries such as trapezoidal, circular, it is totally difficult to find a solution, because the governing equations are implicit. Therefore, the approximate solution could be determined by trial, numerical or graphical methods. These methods tend to take a long time to find an approximate solution, so a simple formula will be more convenient for consultant hydraulic design engineers. The existing formulas are bascially simple, but the relative error between the approximate solutions and true values can reach $9 \%$ or greater. This article presents new explicit regression equations for the critical depth in a partially full circular culvert. The proposed formula is quite simple, and the relative maximum error is $3.03 \%$. It would be very useful as a reference for design in conduit engineering.
\end{abstract}

Keywords: Critical depth, Circular culvert, Froude number, Regression equations, Specific energy section.

(C) 2020 University of Transport and Communications

\section{INTRODUCTION}

Critical depth plays an important role in hydraulics engineering, agricultural irrigation, and sewerage works design. Critical flow is the transition or control flow that possesses the minimum specific energy with a flow rate. When the actual water depth is greater than critical 
depth, subcritical occurs which is mild flow. Conversely, it is supercritical flow which is steep flow [1-4].

Roadway culverts are usually rectangular or circular in cross-section, but circular-shaped culverts are widely used for roadway drainage and agricultural irrigation due to their excellent hydraulic properties and convenient construction.

The governing equations for the critical depth in a circular culvert are complicated implicit equations and not able to find the analytical solutions. The following methods are used for determining the critical depth: trial; numerical and graphical methods [3,9-10], but these methods tend to take a long time to find an approximate solution. Hydraulic engineers need a more simple formula for more convenience in designing consultants. Some existing formulas are simple, but the relative error between the approximate solutions and true values is quite high, can reach $9 \%$ or greater. Therefore, the proposed formula for critical depth in a partially full circular culvert is established using the curve fitting method while considering non-dimensional parameters. The proposed formula would be useful as a reference for design in conduit engineering.

\section{METHODOLOGY}

\subsection{Basic theory of critical depth formula}

Specific energy is the energy at a cross-section of open channel flow with respect to the channel bed. The concept of specific energy is very useful in defining critical water depth and in the analysis of open channel flow [1-3].

$$
E=h+\frac{\alpha v^{2}}{2 g}=h+\frac{\alpha Q^{2}}{2 g A^{2}}
$$

where $\mathrm{E}$ is specific energy at the cross-section $(\mathrm{m}), \mathrm{h}$ is water depth $(\mathrm{m}) ; \alpha$ is the coefficient of kinetic energy (Coriolis coefficient) (-), range value from 1.10 to 1.20 for regular channels [3] (engineers usually assume alpha equal to 1.0 for designing purposes), $g$ is gravitational acceleration, $g=9.81\left(\mathrm{~m} / \mathrm{s}^{2}\right)$; $\mathrm{Q}$ is the flow discharge $\left(\mathrm{m}^{3} / \mathrm{s}\right)$, $\mathrm{A}$ is flow area $\left(\mathrm{m}^{2}\right)$.

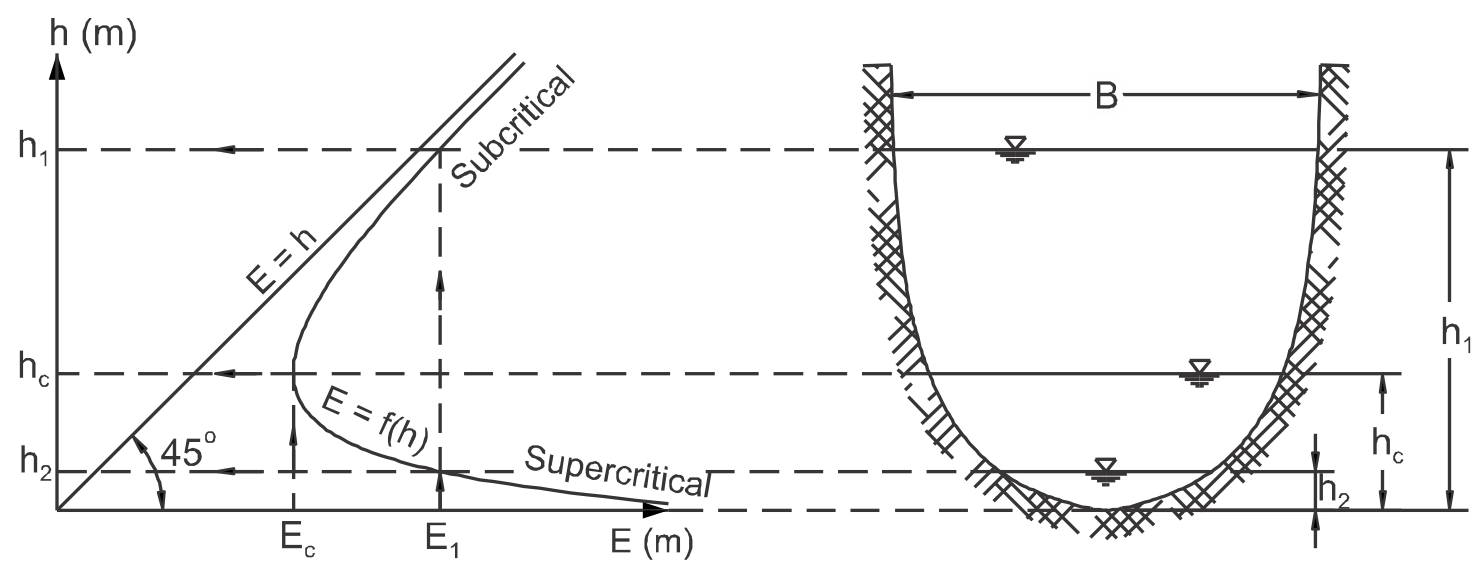

Figure 1. Specific energy diagram.

When the specific energy (E) is minimum, it is known as the critical flow condition and corresponding critical depth $\left(\mathrm{h}_{\mathrm{c}}\right.$ ) (Figure 1). Differentiating eq. (1) with respect to $\mathrm{h}$ (in case $\mathrm{Q}$ $=$ constant ) and equating to zero, the governing critical flow equation is defined. 
Transport and Communications Science Journal, Vol. 71, Issue 7 (09/2020), 840-852

$$
\frac{\alpha Q^{2}}{g A^{3}} B=1 \Leftrightarrow F \mathrm{r}^{2}=1
$$

where B is top width of water surface $(\mathrm{m})$, Fr is Froude number, $\mathrm{Fr}=\frac{v}{\sqrt{g h}}[4,9]$.

On basic of eq. (2), the governing equation for computation of critical depth is described:

$$
\frac{\alpha Q^{2}}{g}=\frac{A^{3}}{B}
$$

The parameters $\alpha, \mathrm{Q}, \mathrm{g}$ are constant, the values of $\mathrm{A}$ and $\mathrm{B}$ depend upon the value of $\mathrm{h}$. If eq. (3) is correct, the critical depth is found.

\subsection{Characteristics of typical channel cross-sections}

The common cross-sections such as rectangular, triangular, trapezoidal and circular are classified according to the following parameters: flow area $(\mathrm{A})$, wetted perimeter $(\mathrm{P})$, hydraulic radius $(\mathrm{R}=\mathrm{A} / \mathrm{P})$ and top width $(\mathrm{B})$. Table 1 summarizes typical open channel flow cross-sections.

Table 1. Open channel geometries.

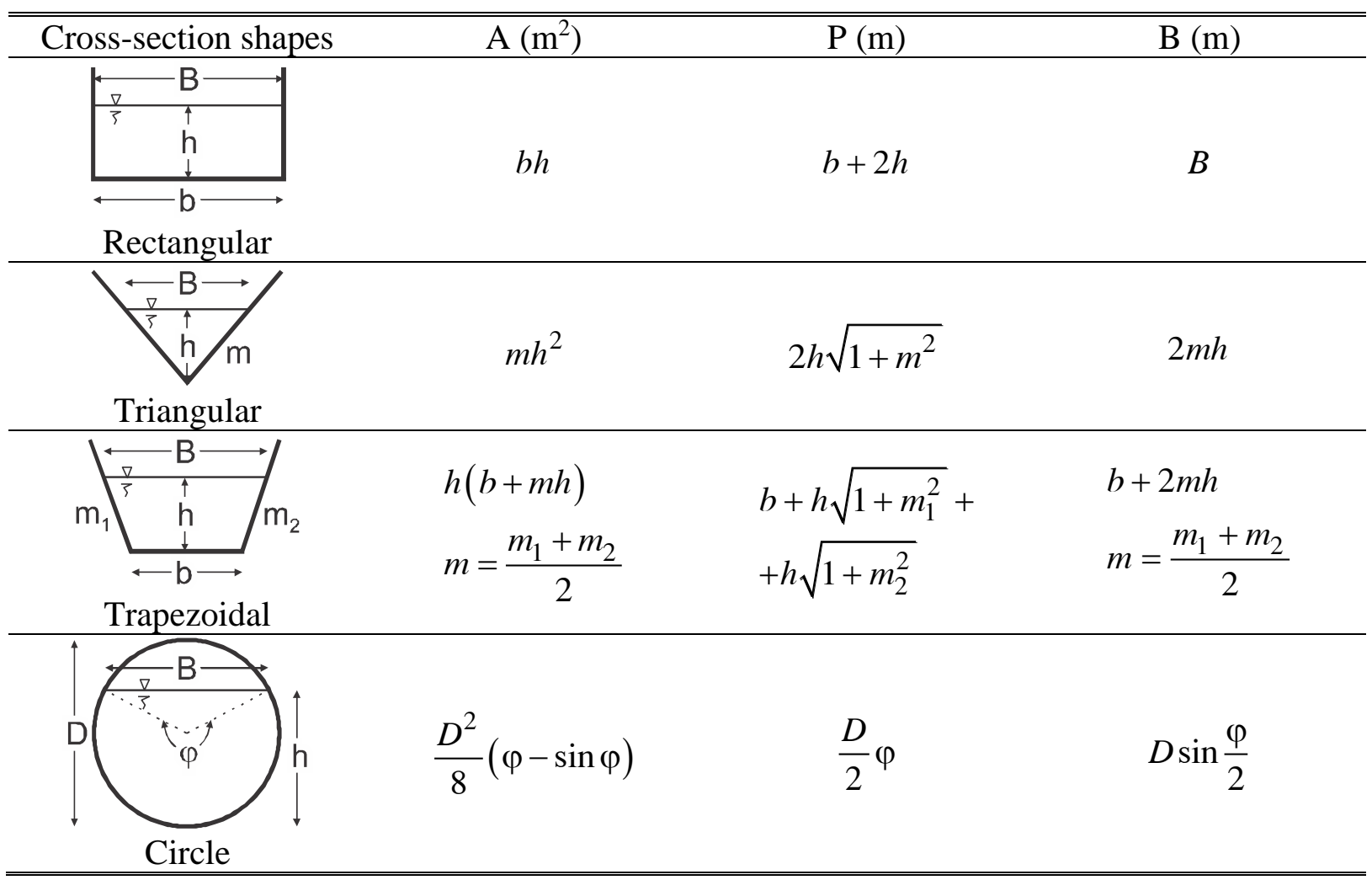

In Table $1, \mathrm{~b}$ is bottom width $(\mathrm{m}), \mathrm{m}$ is side slope [-], $\varphi$ is central angle ( $\mathrm{rad})$ and $\mathrm{D}$ is the culvert diameter.

\subsection{Computation of critical depth}

If cross-section types are rectangular and triangular, the analytical solutions can be found. Substituting for A and B from Table 1 into eq. (3), the critical depth formula is as follows: 
Transport and Communications Science Journal, Vol. 71, Issue 7 (09/2020), 840-852

$$
\begin{aligned}
& h_{c_{\text {rec }}}=\sqrt[3]{\frac{\alpha Q^{2}}{g b^{2}}} \text { (rectangular section) } \\
& h_{c_{t r i}}=\sqrt[5]{\frac{2 \alpha Q^{2}}{g m^{2}}} \text { (triangular section) }
\end{aligned}
$$

However, if cross-section types are trapezoidal and circular, it would be not found the analytical solutions. Hence, the approximate solutions could be determined by trial and graphical methods or using semi-empirical equations for the estimation of critical depth $\mathrm{h}_{\mathrm{c}}$ $[7,8]$.

Hoang Nam Binh [1] proposed Agroskin's critical depth formula form in the trapezoidal open channel with application range $\frac{m h_{c_{r e c}}}{b}=0 \div 4$ :

$$
h_{c_{\text {tra }}}=h_{c_{\text {rec }}}\left(c_{0}+c_{1} \frac{m h_{c_{\mathrm{rec}}}}{b}+c_{2}\left(\frac{m h_{c_{\mathrm{rec}}}}{b}\right)^{2}\right) \text { (trapezoidal section) }
$$

where:

$$
\begin{aligned}
& c_{0}=1.000 ; c_{1}=-\frac{1}{3} ; c_{2}=0.105 \text { in the case of } \frac{m h_{r e c}}{b}=0 \div 1 \\
& c_{0}=0.939 ; c_{1}=-\frac{1}{5} ; c_{2}=0.031 \text { in the case of } \frac{m h_{r e c}}{b}=1 \div 2 \\
& c_{0}=0.878 ; c_{1}=-\frac{2}{15} ; c_{2}=0.013 \text { in the case of } \frac{m h_{c_{r e c}}}{b}=2 \div 3 \\
& c_{0}=0.818 ; c_{1}=-\frac{2}{21} ; c_{2}=0.007 \text { in the case of } \frac{m h_{c_{r e c}}}{b}=3 \div 4
\end{aligned}
$$

There are some semi-empirical equations for the circular section:

$$
\begin{gathered}
h_{c_{\text {ir }}}=\frac{1.01}{D^{0.26}}\left(\frac{\alpha Q^{2}}{g}\right)^{0.25} \quad(\text { Straub [7]) } \\
h_{c_{c \mathrm{ir}}}=D\left(0.77 \frac{g^{3} D^{15}}{Q^{6}}+1\right)^{-0.085} \quad \text { (Swamee [8]) } \\
h_{c_{\text {cir }}}=\frac{0.9584 Q^{0.5}}{\left(1+0.0106 \frac{Q^{0.25} \mathrm{D}^{0.25}}{g^{0.26} \mathrm{D}^{1.30}-0.0132} \frac{Q^{3.726}}{g^{1.863} \mathrm{D}^{9.315}}\right)^{-10.022}} \quad \text { (Vatankhah [10]) } \\
h_{c_{\text {cir }}}=D\left(1+13.6 \frac{g^{2.1135} \mathrm{D}^{10.5675}}{Q^{4.227}}-13 \frac{g^{2.1} \mathrm{D}^{10.5}}{Q^{4.2}}\right)^{-0.1156} \quad \text { (Vatankhah [10]) }
\end{gathered}
$$


Transport and Communications Science Journal, Vol. 71, Issue 7 (09/2020), 840-852

$$
h_{c_{c i \mathrm{r}}}=D\left(1+3.83 \frac{g^{2.1454} \mathrm{D}^{10.727}}{Q^{4.2908}}-3.2 \frac{g^{2.1} \mathrm{D}^{10.5}}{Q^{4.2}}\right)^{-0.115} \quad(\text { Shang [6]) }
$$

Eq. (7) and (8) are quiet simple form of formula. In order to evaluate the accuracy of the formulas, the eq. (7) and (8) were applied by calculating $h_{c_{c i r}}$ with $\mathrm{D}=0.5-4.0 \mathrm{~m}$ and value of discharge $\mathrm{Q}$ so that $\mathrm{h}_{\mathrm{c}} / \mathrm{D}=0.1-0.90$. Substituting these values into eq. (3) and then calculating relative error $\varepsilon(\%)$ by eq. (12):

$$
\varepsilon=\left(\mathrm{LS}_{(3)}-\mathrm{RS}_{(3)}\right) / \mathrm{RS}_{(3)}
$$

where: $\mathrm{LS}_{(3)}$ is left-hand side of eq. (3), $\mathrm{RS}_{(3)}$ is right-hand side of eq. (3).

Error analysis results are shown in Figure 2. The relative error of eq. (7) is divergence and the maximum error is $19.96 \%$. So, eq. (7) does not meet the requirements for culvert design. The maximum relative error of eq. (8) is $5.06 \%$, and suitable application condition is $\mathrm{h}_{\mathrm{c}} \geq \mathrm{D} / 2$.

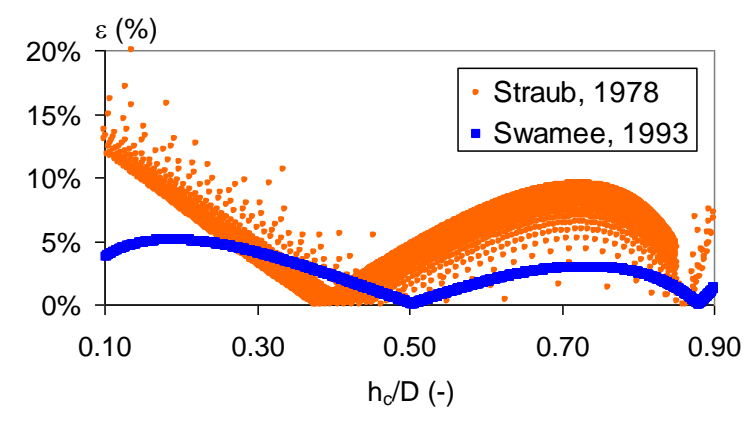

Figure 2. Error analysis of the simple formula type (7) and (8).

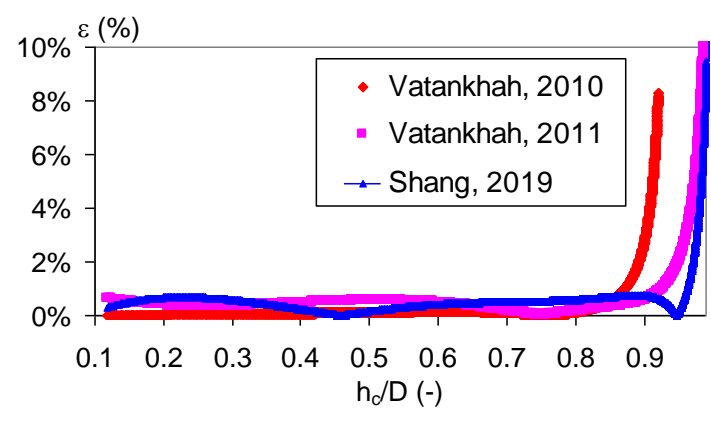

Figure 3. Error analysis of the complex formula type (9), (10) and (11).

Eq. (9), (10) and (11) are the more complex formula forms than eq. (7) and (8), in which eq. (9) is the most complex form with an application range $h_{c} / D \leq 0.92$. Eq. (9) was proposed by Vatankhah in 2010 and then Vatankhah developed a method of calculating the critical depth in a circular cross-section and prosposed a new semi-empirical formula (10) in 2011 [10]. Shang et al [6] applied the PSO algorithm in MATLAB to optimize the value of parameters and proposed the formula (11). Eq. (10) and (11) have the same application range $\mathrm{h}_{\mathrm{c}} / \mathrm{D}<1.00$. When $\mathrm{h}_{\mathrm{c}} / \mathrm{D} \leq 0.85$, the maximun relative errors $(\varepsilon)$ are less than $1 \%$. The highest precision is eq. (11). However, when $h_{c} / D>0.85$ the relative error may be increased and exceed the target error when $h_{c} / D>0.95$ (Figure 3).

\subsection{Method of establishing the formula}

Substituting for A and B from Table 1 with the circular section into eq. (3) to get governing equation of critical depth (13):

$$
\frac{\alpha Q^{2}}{g}=\frac{A^{3}}{B}=\frac{\left(\frac{D^{2}}{8}(\varphi-\sin \varphi)\right)^{3}}{D \sin \frac{\varphi}{2}}
$$


Transport and Communications Science Journal, Vol. 71, Issue 7 (09/2020), 840-852

$$
\Rightarrow \quad \frac{8^{3} \alpha Q^{2}}{g D^{5}}=\frac{(\varphi-\sin \varphi)^{3}}{\sin \frac{\varphi}{2}}
$$

On the other hand, from the circular culverts diagram in Table 1, it is easy to recognize that:

So

$$
\cos \left(\frac{\varphi}{2}\right)=1-2 \frac{h}{D}
$$

and

$$
\begin{aligned}
& \varphi=2 \arccos \left(1-2 \frac{h_{c_{\text {cir }}}}{D}\right) \\
& \frac{h_{c_{\text {ir }}}}{D}=\frac{1}{2}\left(1-\cos \left(\frac{\varphi}{2}\right)\right)
\end{aligned}
$$

\subsubsection{Graphical method}

Given $\mathrm{Q}$ and D, draw a relationship curve between $\mathrm{h}$ and $\mathrm{E}$ (similar to Figure 1) from eq. (1) by changing $\mathrm{h}=\mathrm{h}+\Delta \mathrm{h}$, where $\mathrm{A}$ in eq. (1) is determined by the formula in Table 1 . The extreme point of the curve corresponds to $h$ is the critical depth. The accuracy of the solution depends on the resolution of the curve. The more $\Delta \mathrm{h}$ decreases, the more the resolution of the curve increases, leading to a more accurate solution. However, it would take a long time to calculate. Conversely, the calculation time can be reduced but the approximate solution does not match the actual solution. In addition, if the design plan is changed, the relationship curve must be redrawn according to the new parameter. Therefore, this method is not convenient for consultant hydraulic design engineers.

\subsubsection{Trial and numerical methods}

From the governing equation of critical depth (13), the algorithm to determine critical depth is as follows (Figure 4):

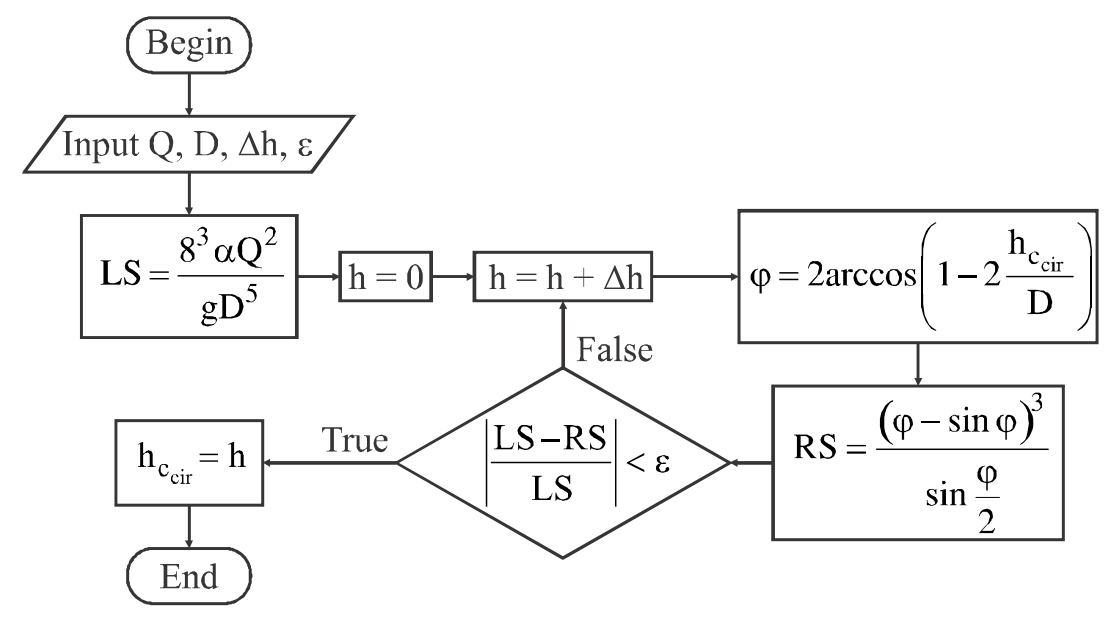

Figure 4. The flow chart to solve the governing equation. 
The growth in computing power has revolutionized the use of realistic mathematical models. Numerical approximation for the governing equation by the algorithm shown in Figure 4 to solve the above equation for $\varphi$ and then for $h_{c}$. The approximate solution obtained by numerical method has a very high accuracy ( $\varepsilon$ could be 0.001 or less), and takes very little time to calculate. However, the coding ability is a major barrier for many consultant hydraulic design engineers.

Users can apply trial method to find critical depth as shown in Table 2.

Table 2. Calculating critical depth by trial method.

\begin{tabular}{cccccc}
\hline \hline $\mathrm{h}(\mathrm{m})$ & $\mathrm{LS}_{(13)}$ & $\varphi(\mathrm{rad})$ & $\mathrm{RS}_{(13)}$ & $\varepsilon$ & Note \\
\hline $\mathrm{h}_{1}$ & $\mathrm{LS}_{(13)}$ & $\varphi_{1}$ & $\left(\mathrm{RS}_{(13))_{1}}\right.$ & $\varepsilon_{1}$ & True/False \\
\hline$\cdots$ & $\cdots$ & $\cdots$ & $\ldots$ & $\ldots$ & $\ldots$ \\
\hline $\mathrm{h}_{\mathrm{n}}$ & $\mathrm{LS}_{(13)}$ & $\varphi_{\mathrm{n}}$ & $\left(\mathrm{RS}_{(13))_{\mathrm{n}}}\right.$ & $\varepsilon_{\mathrm{n}}$ & True/False \\
\hline \hline
\end{tabular}

First, a value of $h$ is assumed and then defining the values $\operatorname{LS}_{(13)}$ by left-hand side of eq. (13), $\varphi$ by eq. (15), $\mathrm{RS}_{(13)}$ by right-hand side of eq. (13) and $\varepsilon=\left(\mathrm{LS}_{(13)}-\mathrm{RS}_{(13)}\right) / \mathrm{RS}_{(13)}$. If $\mathrm{LS}_{(13)}>\mathrm{RS}_{(13)}$ then the value of $\mathrm{h}$ must be increased. Otherwise, the value of $\mathrm{h}$ must be decreased. Until $\varepsilon$ less than or equal target error (defined by the user, usually taken as 0.05 ), then the note column in Table 2 is True and the critical depth is found. The accuracy of solution and calculation time depends on user experience and target error.

\subsubsection{Curve fitting method}

The curve fitting method is the way that a series of data points are modelled or represented by assigning the "best fit" function along with the entire range. The curve fitting method refers to a regression analysis of the relationship between a dependent variable and one or more independent variables. When a relationship is approximated by a straight line, it is called linear regression. When the relationship is a curve form, it is called nonlinear regression or curve fitting. The study used the Microsoft Excel regression analysis tool to find the equation that best fits the data sets by using the "least squares" method. Excel's built-in regression types include linear, polynomial, logarithmic, exponential, and power function.

By equations (13), (12) and (16), there are two relation curves to establish the formula with an application range $0<\frac{h_{c_{c i \mathrm{r}}}}{D}<1.0$ :

$$
\varphi \sim \frac{(\varphi-\sin \varphi)^{3}}{\sin \frac{\varphi}{2}} \Rightarrow \varphi=f\left(\frac{8^{3} \alpha Q^{2}}{g D^{5}}\right)(\text { see Figure 5) }
$$


and

$$
\frac{h_{c_{c \mathrm{ir}}}}{D} \sim \frac{8^{3} \alpha Q^{2}}{g D^{5}} \Rightarrow \frac{h_{c_{c \mathrm{ir}}}}{D}=f\left(\frac{8^{3} \alpha Q^{2}}{g D^{5}}\right) \text { (see Figure 6) }
$$

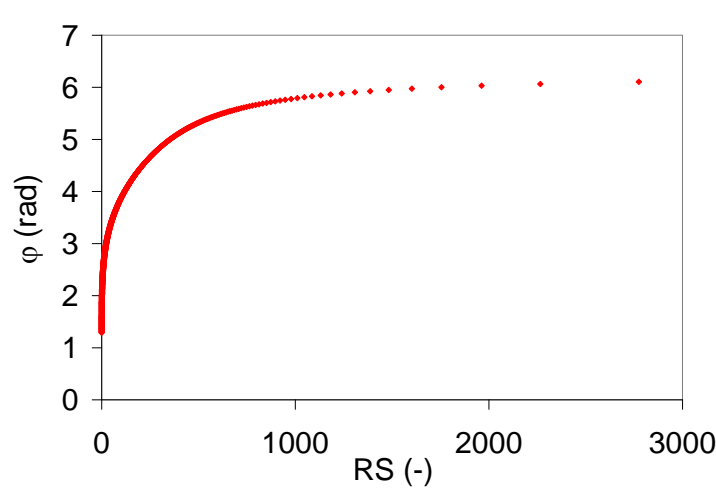

Figure 5. The relation curve between $\varphi$ and right-hand side (RS) of eq. (13).

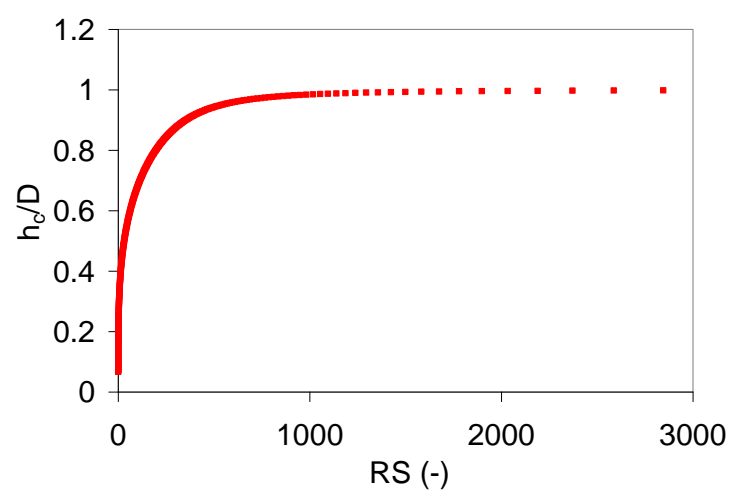

Figure 6. The relation curve between $\mathrm{h}_{d} / \mathrm{D}$ and right-hand side (RS) of eq. (13).

Regression analysis eq. (17) and (18) with curve fitting method, the proposed formula type 1 and type 2 yield the following form:

Type 1:

$$
h_{c_{c \mathrm{ri}}}=F(\cos (Q, D))
$$

Type 2:

$$
h_{c_{\text {cir }}}=F(Q, D)
$$

\section{RESULTS}

\subsection{Formula type 1}

Apply the logarithmic transformation of the independent variable and divide the relation curve eq. (17) into 2 parts, $h_{c} \leq D / 2$ and $h_{c}>D / 2$, the actual curves are shown in Figure $7(\varphi=$ $0 \div \pi)$ and Figure $8(\varphi=\pi \div 2 \pi)$.

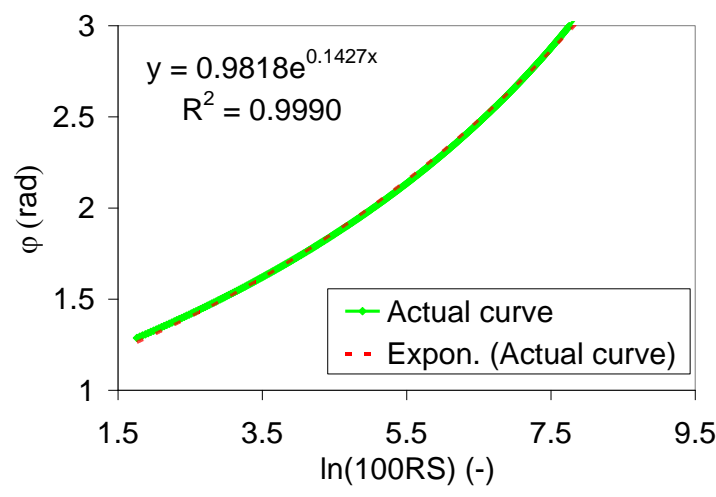

Figure 7. The relation curve between $\varphi$ and logarithmic right-hand side (RS) of eq. (13) with $h_{c} \leq D / 2$.

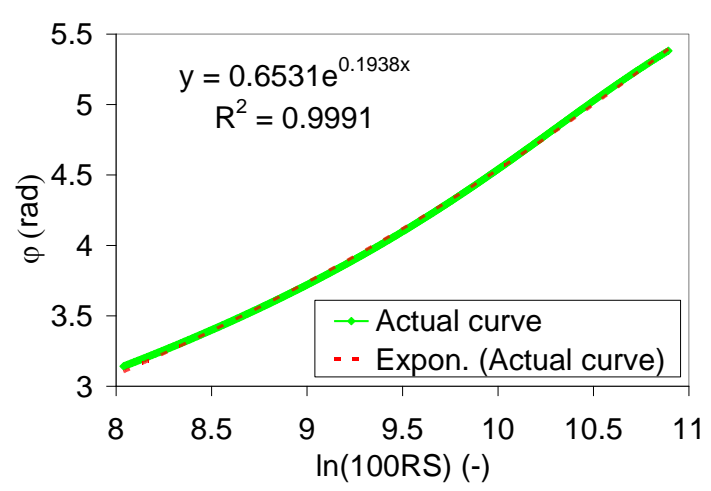

Figure 8. The relation curve between $\varphi$ and logarithmic right-hand side (RS) of eq. (13) with $h_{c}>D / 2$. 
Using curve fitting method, the regression equation of relation curve eq. (17) are established in the exponential function. The R-squared value is approximately 1.0.

$$
\begin{aligned}
& \varphi=0.9818 \exp \left(0.1427 \ln \left(100 \frac{8^{3} \alpha Q^{2}}{g \mathrm{D}^{5}}\right)\right)\left(\text { with } \mathrm{h}_{\mathrm{c}} \leq \mathrm{D} / 2\right) \\
& \varphi=0.6531 \exp \left(0.1938 \ln \left(100 \frac{8^{3} \alpha Q^{2}}{g \mathrm{D}^{5}}\right)\right)\left(\text { with } \mathrm{h}_{\mathrm{c}}>\mathrm{D} / 2\right)
\end{aligned}
$$

The dashed curves in Figure 7 and Figure 8 draw trendline eqs. (21) and (22) respectively. Substituting for $\varphi$ from eqs. (21) and (22) into eq. (16) the formula type 1 to calculate the critical depth in the circular culvert is obtained.

$$
h_{c_{\text {cir }}}=\frac{D}{2}-\frac{D}{2} \cos \left(c_{1} \frac{Q^{c_{2}}}{D^{c_{3}}}\right)
$$

where $c_{1}, c_{2}$ and $c_{3}$ are constants

$$
\begin{aligned}
& \text { If } \mathrm{h}_{\mathrm{c}} \leq \mathrm{D} / 2 \text { then } \mathrm{c}_{1}=1.6654, \mathrm{c}_{2}=0.2854 \text { and } \mathrm{c}_{3}=0.7135 \\
& \text { If } \mathrm{h}_{\mathrm{c}}>\mathrm{D} / 2 \text { then } \mathrm{c}_{1}=1.7161, \mathrm{c}_{2}=0.3876 \text { and } \mathrm{c}_{3}=0.9691
\end{aligned}
$$

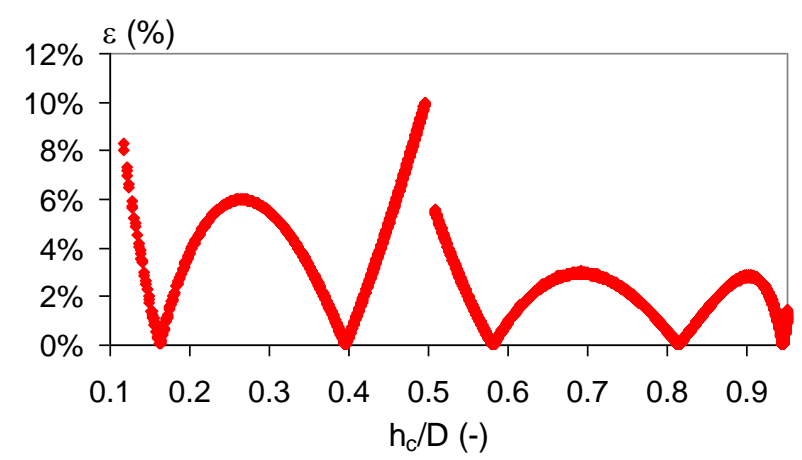

Figure 9. Error analysis of the critical depth formula type 1.

The relative error of eq. (23) is shown in Figure 9. The maximum relative error is $10.5 \%$. The suitable application condition is $0.55 \leq \mathrm{h}_{\mathrm{c}} / \mathrm{D} \leq 0.95$ and the maximum error in the application range is $3.05 \%$.

\subsection{Formula type 2}

Relation curve eq. (18) is drawn by changing $\varphi$. The left-hand side (LS) of eq. (18) is calculated by eq. (16) and the right-hand side (RS) of eq. (18) is calculated by eq. (13). This relation curve is shown in Figure 10 (actual curve), in which, the horizontal axis value is $\ln (100 \mathrm{RS})$. 
Transport and Communications Science Journal, Vol. 71, Issue 7 (09/2020), 840-852

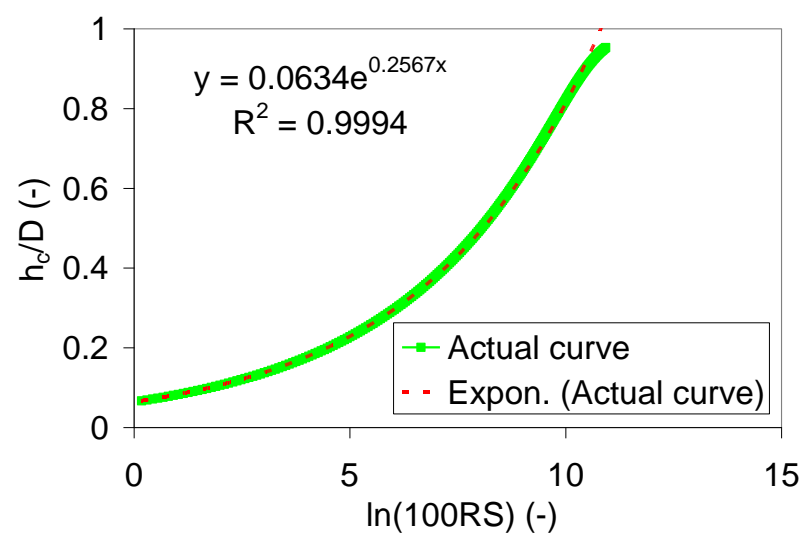

Figure 10. The relation curve between $\varphi$ and logarithmic right-hand side (RS) of eq. (13).

Calculating the least squares fit through points, the regression equation of relation curve eq. (18) is obtained

$$
\begin{aligned}
& \frac{h_{c_{\text {ir }}}}{D}=0.0634 \exp \left(0.2567 \ln \left(100 \frac{8^{3} \alpha Q^{2}}{g \mathrm{D}^{5}}\right)\right) \\
\Rightarrow \quad \frac{h_{c_{\text {cir }}}}{D} & =0.0634\left(100 \frac{8^{3} \alpha Q^{2}}{g \mathrm{D}^{5}}\right)^{0.2567}
\end{aligned}
$$

Transforming eq. (24), the formula type 2 to calculate the critical depth in circular culvert yields the following form eq. (25):

$$
h_{c_{\text {cir }}}=0.5697 \frac{Q^{0.5126}}{\mathrm{D}^{0.2815}}
$$

The relative error of eq. (25) is shown in Figure 11. If $h_{d} / D \leq 0.80$, the maximum relative error is $3.03 \%$. When $h_{c} / D>0.80$, the error increases quite quickly. Thus, eq. (25) is suitable for application within $\mathrm{h}_{\mathrm{c}} / \mathrm{D} \leq 0.80$.

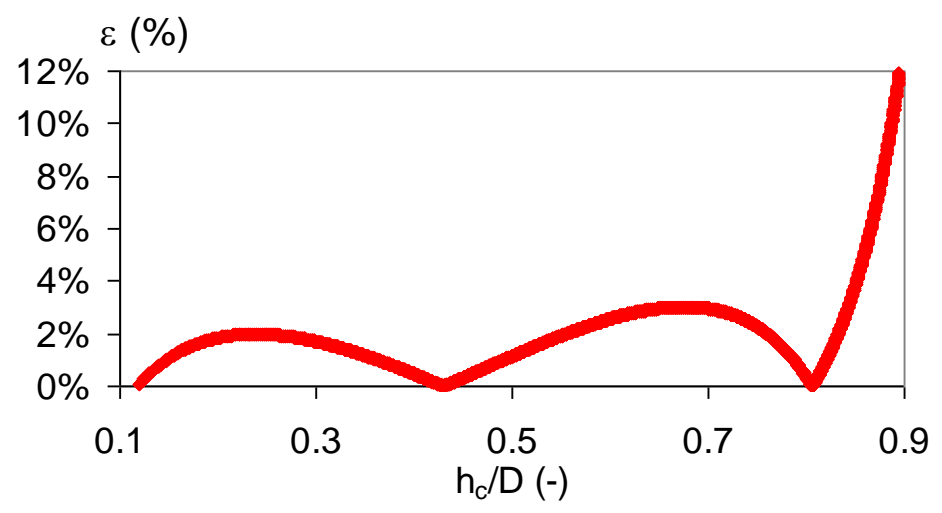

Figure 11. Error analysis of the critical depth formula type 2. 
Transport and Communications Science Journal, Vol. 71, Issue 7 (09/2020), 840-852

In order to expand application range, the proposed formula eq. (26) uses both eq. (23) and (25). The procedure for using eq. (26) is shown in Figure 12.

$$
\left\{\begin{array}{l}
\left.h_{c_{\text {cir }}}=0.5697 \frac{Q^{0.5126}}{\mathrm{D}^{0.2815}} \quad \text { (in the case of } 0.05<\frac{h_{c}}{D} \leq 0.80\right) \\
h_{c_{\text {cir }}}=\frac{D}{2}-\frac{D}{2} \cos \left(1.7161 \frac{Q^{0.3876}}{D^{0.9691}}\right) \quad\left(\text { in the case of } 0.80<\frac{h_{c}}{D} \leq 0.95\right)
\end{array}\right.
$$

From Figure 12, it could be seen that the critical depth in circular culvert is calculated by eq. (25), if $\frac{h_{c}}{D} \leq 0.8$ then the critical depth is found, otherwise it is calculated by eq. (23). The error analysis of the proposed formula is shown in Figure 13.

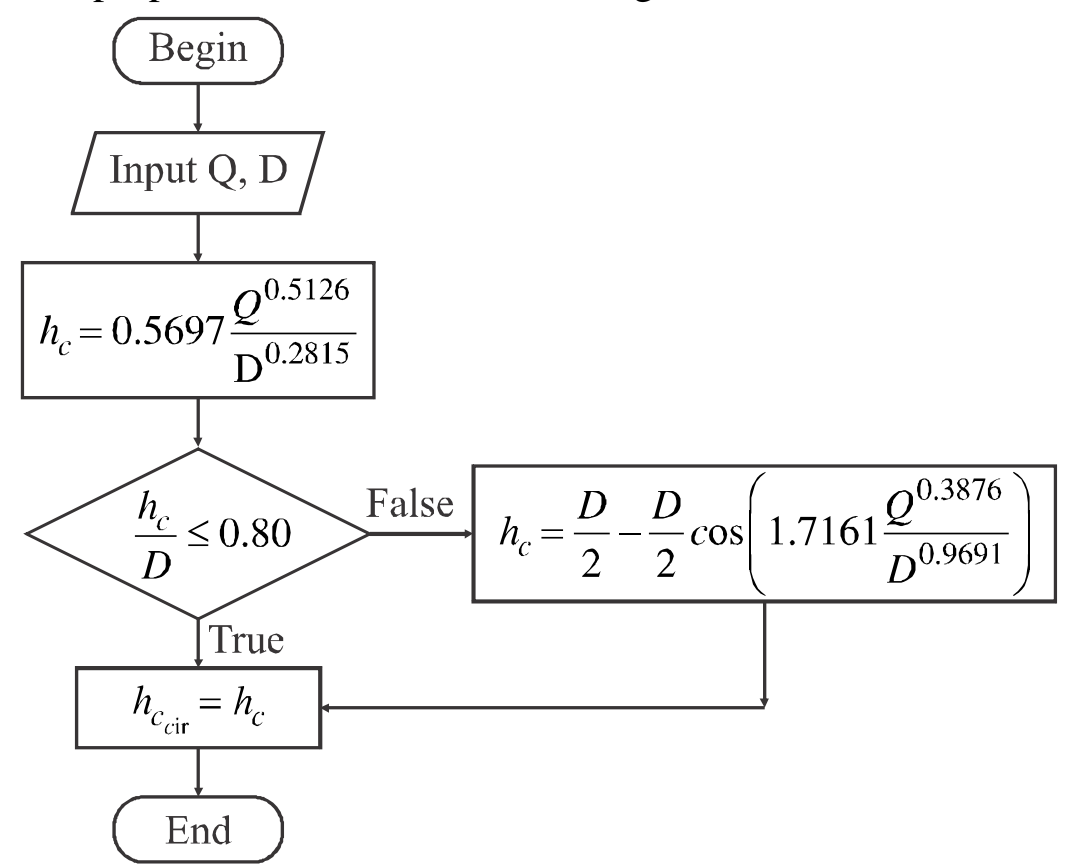

Figure 12. The critical depth determination algorithm applies to proposed formula.

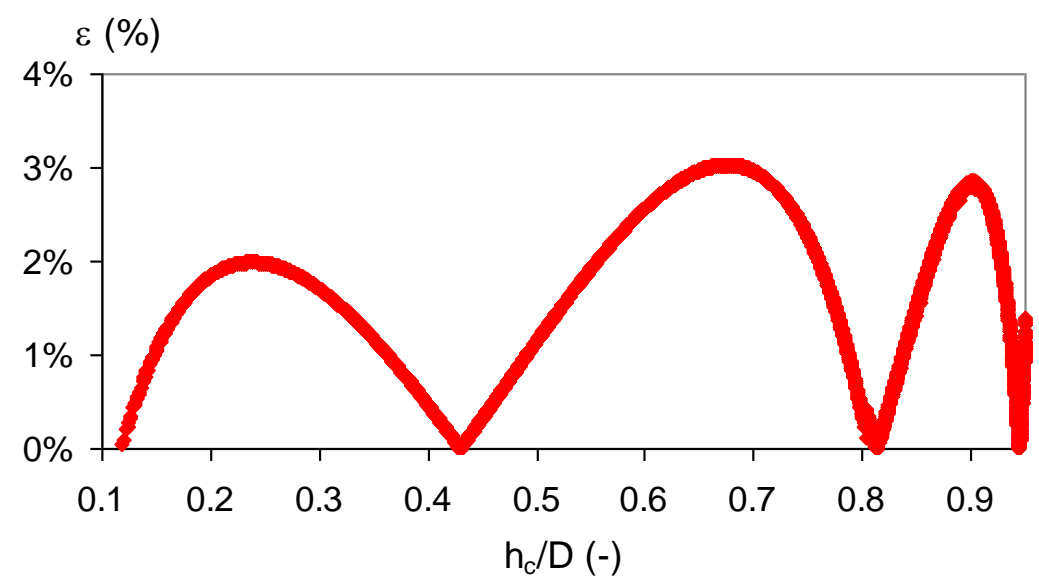

Figure 13. Error analysis of the proposed critical depth formula for circular section. 
Transport and Communications Science Journal, Vol. 71, Issue 7 (09/2020), 840-852

Table 3. Comparing proposed formula to others.

\begin{tabular}{cccc}
\hline \hline Author's formula & Maximum relative error (\%) & Application range & Note \\
\hline Straub [7] & $19.96 \%$ (Figure 2) & $\mathrm{h}_{\mathrm{d}} / \mathrm{D} \leq 0.85$ & eq. (7) \\
\hline Swamee [8] & $5.06 \%$ (Figure 2) & $\mathrm{h}_{\mathcal{C}} / \mathrm{D} \leq 0.90$ & eq. (8) \\
\hline Proposed & $3.03 \%$ (Figure 13) & $\mathrm{h}_{\mathcal{c}} / \mathrm{D} \leq 0.95$ & eq. (26) \\
\hline \hline
\end{tabular}

The three formulas to calculate the critical depth in circular culvert, which are shown in Table 3. Eq. (7) have the maximum relative error that can reach to $20 \%$. The accuracy of eq. (8) is higher than eq. (7) with an application range $h_{c} / D \leq 0.90$. However, in the case of $h_{c} / D$ $=0.95$, the maximum relative error of eq. (8) can exceed $9 \%$. For example, if $\mathrm{Q}=1.55 \mathrm{~m}^{3} / \mathrm{s}$ and $\mathrm{D}=0.75 \mathrm{~m}$ then $\mathrm{h}_{\mathrm{c}}=0.717 \mathrm{~m}, \mathrm{~h}_{\mathrm{c}} / \mathrm{D}=0.95$ and $\varepsilon=9.05 \%$.

From Figure 13, it could be seen that the application range of the proposed formula in this article is $h_{c} / D \leq 0.95$ and the maximum relative error is $3.03 \%$. In general, the proposed formula has the ability to determine the critical depth in a circular culvert with higher accuracy and wider application range than Straub's and Swamee's formulas.

\section{CONCLUSION}

Critical depth is an important parameter in culvert designing and it is normally determined by trial and graphical methods for circular sections. However, these methods often take a long time to calculate and can cause errors that exceed the target error. The semiempirical equations are more convenient for designers, but the accuracy of those does not meet the requirements in hydraulic engineering. This article presents the governing equation for computation of the critical depth and the curve fitting method to establish proposed formula. The eq. (26) is a quite simple formula. The critical depth in the circular culvert can be easy computed in practical with practical range of depth $h_{c} / D \leq 0.95$ and maximum percentage error less than the target error for design in conduit engineering.

\section{REFERENCES}

[1]. Hoang Nam Binh, Expanding the application range of Agroskin's critical depth formula in trapezoidal open channel, Transport and Communications science journal, 67 (2018), 17-23.

[2]. Nguyen Canh Cam, Vu Van Tao, Hydraulics, Vol. 1, Agricultural Publishing House, 2006, Vietnamese.

[3]. Chou V. T., Open-channel hydraulics, McGraw-Hill book company, 1988, ISBN 07-010776-9.

[4]. Hanif C. M., Open-Channel Flow, Second Edition, Springer Science+Business Media, LLC, 2008, ISBN 978-0-387-30174-7.

[5]. MIDUSS Version 2 Reference Manual, Hydraulic Theory, Alan A. Smith Inc, http://miduss.com/theory-Pipe-Design.htm

[6]. Haixin Shang, Song Xu, Kuandi Zhang, Luyou Zhao, Explicit solution for critical depth in closed conduits flowing partly full, Water, 11 (2019) 2124. https://doi.org/10.3390/w11102124 
Transport and Communications Science Journal, Vol. 71, Issue 7 (09/2020), 840-852

[7]. Straub WO., A quick and easy way to calculate critical and conjugate depths in circular open channels. Civil Engineering, 1978.

[8]. Swamee PK, Critical depth equations for irrigation canals, Journal of Irrigation and Drainage Engineering, 119 (1993) 400-9. https://doi.org/10.1061/(ASCE)0733-9437(1993)119:2(400)

[9]. U.S. Department of Transportation, Hydraulic design of highway culverts, Third edition, Publication No. FHWA-HIF-12-026, 2012.

[10].Vatankhah A. R., Easa S. M., Explicit solutions for critical and normal depths in channels with different shapes, Flow Measurement and Instrumentation, 22 (2011) 43-49, https://doi.org/10.1016/j.flowmeasinst.2010.12.003

[11].Vyzgo M. S., Determining critical depth of waterways, canals, Gidrotekhnika i Melioratsiya, 2, (1949), Moscow.

[12].Yerzhanova N. K. et al., Critical section and critical depth in open flows finding device, Magazine of Civil Engineering, 8 (2017) 106-114. https://doi.org/10.18720/MCE.76.10 